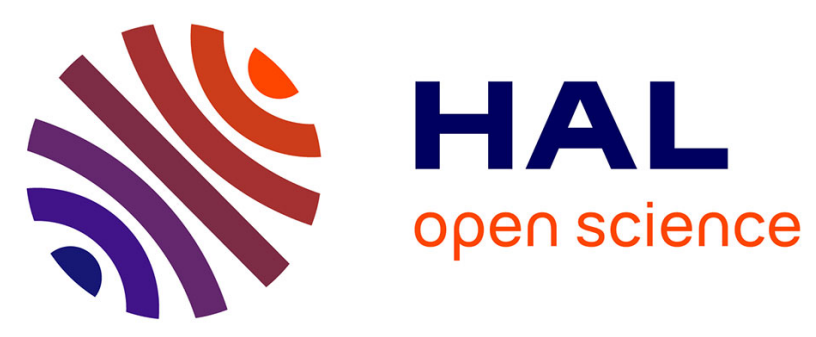

\title{
A new fully two-dimensional conservative semi-Lagrangian method: applications on polar grids, from diocotron instability to ITG turbulence
}

Nicolas Crouseilles, Pierre Glanc, Sever Adrian Hirstoaga, Eric Madaule, Michel Mehrenberger, Jérôme Pétri

\section{To cite this version:}

Nicolas Crouseilles, Pierre Glanc, Sever Adrian Hirstoaga, Eric Madaule, Michel Mehrenberger, et al.. A new fully two-dimensional conservative semi-Lagrangian method: applications on polar grids, from diocotron instability to ITG turbulence. The European Physical Journal D : Atomic, molecular, optical and plasma physics, 2014, 68 (9), DOI: 10.1140/epjd/e2014-50180-9. 10.1140/epjd/e2014-50180-9 . hal-00977342v2

\author{
HAL Id: hal-00977342 \\ https://hal.science/hal-00977342v2
}

Submitted on 15 Dec 2014

HAL is a multi-disciplinary open access archive for the deposit and dissemination of scientific research documents, whether they are published or not. The documents may come from teaching and research institutions in France or abroad, or from public or private research centers.
L'archive ouverte pluridisciplinaire HAL, est destinée au dépôt et à la diffusion de documents scientifiques de niveau recherche, publiés ou non, émanant des établissements d'enseignement et de recherche français ou étrangers, des laboratoires publics ou privés. 


\title{
A new fully two-dimensional conservative semi-Lagrangian method: applications on polar grids, from diocotron instability to ITG turbulence
}

\author{
Nicolas Crouseilles ${ }^{1}$, Pierre Glanc ${ }^{2}$, Sever A. Hirstoaga ${ }^{2}$, Eric Madaule ${ }^{3}$, Michel Mehrenberger ${ }^{2,4}$ and Jérôme Pétri $^{5}$ \\ 1 Inria-Rennes (IPSO team) \& IRMAR, Rennes, France \\ 2 Inria-Nancy (TONUS team) \& IRMA, Strasbourg, France \\ 3 Institut Jean Lamour, Nancy, France \\ 4 Max-Planck-Institut für Plasmaphysik, Garching, Germany \\ ${ }^{5}$ Observatoire Astronomique de Strasbourg, Strasbourg, France
}

Received: date / Revised version: date

\begin{abstract}
While developing a new semi-Lagrangian solver, the gap between a linear Landau run in $1 D \times 1 D$ and a $5 D$ gyrokinetic simulation in toroidal geometry is quite huge. Intermediate test cases are welcome for testing the code. A new fully two-dimensional conservative semi-Lagrangian (CSL) method is presented here and is validated on $2 D$ polar geometries. We consider here as building block, a $2 D$ guiding-center type equation on an annulus and apply it on two test cases. First, we revisit a $2 D$ test case previously done with a PIC approach [18] and detail the boundary conditions. Second, we consider a $4 D$ drift-kinetic slab simulation (see [10]). In both cases, the new method appears to be a good alternative to deal with this type of models since it improves the lack of mass conservation of the standard semi-Lagrangian (BSL) method.
\end{abstract}

PACS. PACS-key 52.30.GzGyrokinetics - PACS-key 52.65.FfPlasma simulation: Fokker-Planck and Vlasov equation

\section{Introduction}

Kinetic models are known to be a precise tool able to describe plasma arising in a tokamak or a beam. However, this accurate description requires an important effort in terms of numerics, since the solution of a kinetic model depends not only on the space variable, but also on the velocity variable, which gives a six dimensional phase space. In addition to the filamentation phenomena and presence of small scales, the numerical approximation of the distribution function solution to the kinetic model is a huge challenge despite the current advancement in computers power.

In recent decades, several different numerical methods have been developed to this aim. Among them, the Particle In Cell (PIC) exploits the Lagrangian formulation of the kinetic equation by solving the characteristics equations. In spite of its relative low computational cost, these kinds of method suffer from inaccuracy in low density regions and low convergence rate when the number of macro-particles increases (see [1]). On the other hand, Eulerian methods have been introduced. They are based on methods like finite volumes or finite difference methods which have been mostly developed in fluids mechanics; the main particularity is that they require a grid of the full phase space which makes them accurate indepen- dently of the value of the distribution function, but they are very costly in terms of memory and computational power. Moreover, a restriction on the time step has to be satisfied for stability reasons. An interesting compromise is the semi-Lagrangian method which tries to take benefit from the Lagrangian approach by tracking the time evolution of the characteristic equations of the kinetic model, and from the Eulerian framework by using a grid of the phase space. In particular, the so-obtained method is free from standard CFL conditions on the time step.

Different semi-Lagrangian methods have been developed over this last 40 years [8], [2], [20], to first compute the solution of the $1 D \times 1 D$ Vlasov-Poisson equation and then of the guiding-center model and more recently of the $5 D$ gyrokinetic model. In addition to the high dimensionality, the spatial geometry of the latter case is toroidal, which makes it more complicated to deal with.

While developing a new semi-Lagrangian solver, the gap between a linear Landau run obtained with the $1 D \times$ $1 D$ Vlasov-Poisson model and a $5 D$ gyrokinetic simulation in toroidal geometry is quite huge. Then, intermediate test cases are welcome for checking the performance and precision of the codes.

Tests for which the analytical solution is known at all times do not include usually sufficient complexity to tackle 
the difficulties of the final problem. Moreover, although interesting, some test cases are not published and often different parameter settings are chosen which makes cross validations with different schemes difficult. Therefore, one purpose of this work is to provide precise parameter settings for two test cases which have intermediate complexity between the analytic test and the realistic physical configuration. This enables us to validate the new conservative semi-Lagrangian method.

We consider here as a building block, a $2 D$ guidingcenter type equation on an annulus satisfied by $f(t, r, \theta)$ :

$$
\partial_{t} f-\frac{\partial_{\theta} \Phi}{r} \partial_{r} f+\frac{\partial_{r} \Phi}{r} \partial_{\theta} f=0, t \in[0, T]
$$

with $(r, \theta) \in \Omega=\left[r_{\min }, r_{\max }\right] \times[0,2 \pi]$, where the potential $\Phi$ solves a Poisson type equation. This model constitutes the basis of the two tests. We first revisit a $2 D$ test case previously done with a PIC approach [18] and detail the boundary conditions. We then consider a $4 D$ drift-kinetic slab simulation [10], [4] which can be viewed as a polar guiding-center equation of type (1) coupled with a Vlasov type equation in the parallel $(z, v)$ direction.

In these two configurations, some first results of a new conservative semi-Lagrangian method are given and compared to the standard one [10]. Indeed, it is well known that this standard method, namely backward semi-Lagrangian (BSL), does not preserve the total mass exactly in the $2 D$ general configuration. Then, in the spirit of [6], we propose a new fully two-dimensional conservative semi-Lagrangian (CSL) method. More precisely, a generalization to the $2 D$ case of [6] is presented in this work to deal with model of the form (1) without considering directional splitting. Indeed, even if time splitting techniques are very efficient in the Vlasov-Poisson case, for the drift-kinetic or guidingcenter models considered here, the characteristic curves are more complicated and need to be tackled in the full $2 D$ case. Hence, to overcome the lack of conservation of the standard BSL method, it seems interesting to develop, validate and compare a $2 D$ conservative semi-Lagrangian method. Let us remark that similar motivations are considered in [21].

The rest of this work is organized as follows: first, the new conservative semi-Lagrangian method is presented. Then, we present in detail two test cases enabling validation of our new method. In section 3, the diocotron instability test case [18] is revisited by detailing the radial boundary conditions and the associated instability rates. Finally section 4 is devoted to a $4 D$ drift-kinetic test case.

\section{A new $2 D$ conservative method}

In this section, we present the new conservative semiLagrangian method (CSL) in the cartesian case. We also briefly recall the standard BSL method and present how the new method can be extended to polar geometries.

We then consider a $2 D$ advection equation of the form

$$
\partial_{t} f+U(x, y) \cdot \nabla_{x, y} f=0,(x, y) \in\left[0, L_{x}\right] \times\left[0, L_{y}\right],
$$

where the divergence free vector field is

$$
U(x, y)=\left(U_{x}(x, y), U_{y}(x, y)\right)
$$

and we consider an initial condition $f(t=0, x, y)=f_{0}(x, y)$. We first define a spatial uniform mesh $x_{i}=i \Delta x, \Delta x=$ $L_{x} / N_{x}, y_{j}=j \Delta y, \Delta y=L_{y} / N_{y}$, and a time discretization $t^{n}=n \Delta t$ with $n \in \mathbb{N}, \Delta t>0$. Then, for all $\left(x_{i}, y_{j}\right)$, we define the characteristics equation with $X(t):=(x, y)(t)$

$$
\dot{X}(t)=U(X(t)), \quad X\left(t^{n+1}\right)=\left(x_{i}, y_{j}\right), \quad t \in\left[t^{n}, t^{n+1}\right] .
$$

Let us denote by $X\left(s ; t^{n+1},\left(x_{i}, y_{j}\right)\right)$ the solution of this differential system, for $s \in\left[t^{n}, t^{n+1}\right]$.

In this subsection, a new conservative semi-Lagrangian is proposed. As in the finite volumes context, the unknowns in this case are not pointwise values but cell average values. Let us introduce a control volume $A_{i, j}=$ $\left[x_{i-1 / 2}, x_{i+1 / 2}\right] \times\left[y_{j-1 / 2}, y_{j+1 / 2}\right]$, with $x_{i \pm 1 / 2}=x_{i} \pm \Delta x / 2$ and $y_{j \pm 1 / 2}=y_{j} \pm \Delta y / 2$ we define the unknown as

$$
\bar{f}(t)_{i, j}=\frac{1}{\Delta x \Delta y} \int_{A_{i, j}} f(t, x, y) d x d y .
$$

We use the fact that the volume is preserved with time. In particular, between times $t^{n}$ and $t^{n+1}$, we get

$$
\begin{aligned}
\left(\bar{f}_{i, j}^{n+1}=\right) & \frac{1}{\Delta x \Delta y} \int_{A_{i, j}} f^{n+1}(x, y) d x d y \\
& =\frac{1}{\Delta x \Delta y} \int_{X\left(t^{n} ; t^{n+1}, A_{i, j}\right)} f^{n}(x, y) d x d y
\end{aligned}
$$

where $X\left(t^{n} ; t^{n+1}, A_{i, j}\right)=\left\{X\left(t^{n} ; t^{n+1},(x, y)\right),(x, y) \in A_{i, j}\right\}$, $X\left(t^{n} ; t^{n+1},(x, y)\right)$ is the solution at time $t^{n}$ of the following characteristics equation

$\dot{X}(t)=U(X(t)), \quad X\left(t^{n+1}\right)=(x, y) \in A_{i, j}, \quad t \in\left[t^{n}, t^{n+1}\right]$.

Since $\bar{f}_{i, j}^{n}$ is known $\forall i, j$ of the mesh, we can reconstruct a piecewise polynomial function $\mathcal{P}_{i, j}$; an example can be the Lagrange reconstruction which satisfies, $\forall i, j$

$\frac{1}{\Delta x \Delta y} \int_{A_{i+\ell, j+k}} \mathcal{P}_{i, j}(x, y) d x d y=\bar{f}_{i+\ell, j+k}^{n}, \forall \ell, k=-d, \ldots, d$,

where $d$ determines the order of the reconstruction $\left(\mathcal{P}_{i, j}\right.$ is a piecewise polynomial function of degree $2 d$ in this case). Another example of reconstruction we propose in this work is based on Hermite reconstruction. Let us introduce some notation. First, we introduce edge average values

$$
\begin{aligned}
& f_{i^{ \pm}, j}^{n} \simeq \frac{1}{\Delta y} \int_{y_{j-1 / 2}}^{y_{j+1 / 2}} f^{n}\left(x_{i \pm 1 / 2}, y\right) d y \\
& f_{i, j^{ \pm}}^{n} \simeq \frac{1}{\Delta x} \int_{x_{i-1 / 2}}^{x_{i+1 / 2}} f^{n}\left(x, y_{j \pm 1 / 2}\right) d x
\end{aligned}
$$

and pointwise values

$$
f_{i^{ \pm}, j^{ \pm}}^{n} \simeq f^{n}\left(x_{i \pm 1 / 2}, y_{j \pm 1 / 2}\right) .
$$


We need to express this quantity as a function of the known values $\bar{f}_{i, j}^{n}$. For this, we first compute the edge values of the form

$$
f_{i \pm, j}^{n}=\sum_{\ell=r^{ \pm}}^{s^{ \pm}} a_{\ell}^{ \pm} \bar{f}_{i+\ell, j}^{n}, f_{i, j^{ \pm}}=\sum_{\ell=r^{ \pm}}^{s^{ \pm}} a_{\ell}^{ \pm} \bar{f}_{i, j+\ell}^{n},
$$

and then the pointwise values

$$
f_{i^{ \pm}, j^{ \pm}}^{n}=\sum_{\ell=r^{ \pm}}^{s^{ \pm}} a_{\ell}^{ \pm} \bar{f}_{i+\ell, j^{ \pm}}^{n}=\sum_{\ell=r^{ \pm}}^{s^{ \pm}} a_{\ell}^{ \pm} \bar{f}_{i^{ \pm}, j+\ell}^{n},
$$

with suitable coefficients $a_{\ell}^{ \pm}, \ell=r^{ \pm}, \ldots, s^{ \pm}$. The integers $r^{ \pm}, s^{ \pm} \in \mathbb{Z}$ are generally linked to the degree of approximation.

Now, for given values of $r^{-} \leq 0, s^{-} \geq 0$, we can compute coefficients $a_{\ell}^{-}, \ell=r^{-}, \ldots, s^{-}$in the following way. We take

$$
a_{\ell}^{-}=-\sum_{k=r^{-}}^{\ell} \frac{\prod_{j=r^{-}, j \notin\{0, k\}}^{s^{-}+1}(-j)}{\prod_{j=r^{-}, j \neq k}^{s^{-}+1}(k-j)}, \ell=r^{-}, \ldots,-1,
$$

and

$$
a_{\ell}^{-}=\sum_{k=\ell+1}^{s^{-}+1} \frac{\prod_{j=r^{-}, j \notin\{0, k\}}^{s^{-}+1}(-j)}{\prod_{j=r^{-}, j \neq k}^{s^{-}+1}(k-j)}, \ell=0, \ldots, s^{-} .
$$

In a similar way, for given values of $r^{+} \leq 0, s^{+} \geq 0$, the coefficients $a_{\ell}^{+}$are given by (for $\ell=r^{+}, \ldots, s^{+}$)

$$
a_{\ell}^{+}=-\sum_{k=r^{+}-1}^{\ell-1} \frac{\prod_{j=r^{+}-1, j \notin\{0, k\}}^{s^{+}}(-j)}{\prod_{j=r^{+}-1, j \neq k}^{s^{+}}(k-j)}, \ell=r^{+}, \ldots, 0,
$$

and

$$
a_{\ell}^{+}=\sum_{k=\ell}^{s^{+}} \frac{\prod_{j=r^{+}-1, j \notin\{0, k\}}^{s^{+}}(-j)}{\prod_{j=r^{+}-1, j \neq k}^{s^{+}}(k-j)}, \ell=1, \ldots, s^{+} .
$$

For $p \in \mathbb{N}^{*}$, we will take

$$
r^{-}=-\left\lfloor\frac{p}{2}\right\rfloor, r^{+}=-\left\lfloor\frac{p-1}{2}\right\rfloor, s^{-}=\left\lfloor\frac{p-1}{2}\right\rfloor, s^{+}=\left\lfloor\frac{p}{2}\right\rfloor .
$$

Using all these expressions, we then can have an approximation

$f_{i, j}^{n}(u, v) \simeq f^{n}\left(x_{i-1 / 2}+u \Delta x, y_{j-1 / 2}+v \Delta y\right)$, for $0 \leq u, v \leq 1$.

For this, we can compute intermediate values

$$
\begin{aligned}
f_{i}^{n}\left(u, j^{ \pm}\right) & \simeq f^{n}\left(x_{i-1 / 2}+u \Delta x, y_{j \pm 1 / 2}\right), \\
f_{i}^{n}(u, j) & \simeq \frac{1}{\Delta y} \int_{y_{j-1 / 2}}^{y_{j+1 / 2}} f^{n}\left(x_{i-1 / 2}+u \Delta x, y\right) d y,
\end{aligned}
$$

which reads, for $k \in\left\{j^{ \pm}, j\right\}$

$$
\begin{aligned}
& f_{i}^{n}(u, k)= \\
& f_{i^{-}, k}^{n}+u\left(f_{i^{+}, k}^{n}-f_{i^{-}, k}^{n}\right)+u(1-u)\left(6 f_{i, k}^{n}-3\left(f_{i^{+}, k}^{n}+f_{i^{-}, k}^{n}\right)\right) .
\end{aligned}
$$

We then obtain the $2 D$ reconstruction for $0 \leq u, v \leq 1$

$$
\begin{aligned}
& f_{i, j}^{n}(u, v)= \\
& f_{i}^{n}\left(u, j^{-}\right)+v\left(f_{i}^{n}\left(u, j^{+}\right)-f_{i}^{n}\left(u, j^{-}\right)\right) \\
& +v(1-v)\left(6 f_{i}^{n}(u, j)-3\left(f_{i}^{n}\left(u, j^{+}\right)+f_{i}^{n}\left(u, j^{-}\right)\right)\right) .
\end{aligned}
$$

Let us remark that we start to reconstruct in the $x$ direction but it is possible to first reconstruct in the $y$ direction by defining $f_{j}^{n}(k, v), k \in\left\{i^{ \pm}, i\right\}$ (which is the counterpart of $\left.f_{i}^{n}(u, k), k \in\left\{j^{ \pm}, j\right\}\right)$.

For each choice of $p$, a specific Hermite method is deduced. Among them, we distinguish odd and even values of $p$. In (6), we can observe that if $p$ is odd, $r^{ \pm}=-s^{ \pm}$so that the reconstruction is continuous: this corresponds to PPM type reconstruction (see [3], [6]). If $p$ is even, the reconstruction is no longer continuous since $r^{ \pm} \neq-s^{ \pm}$: this corresponds to LAGH type reconstruction. We refer to $[9],[5]$ for more details.

The computation of $X\left(t^{n} ; t^{n+1}, A_{i, j}\right)$ needs some details since it is not possible to compute exactly this volume. In practice, we only compute the feet of the characteristics ending at the 4 corners of the cell $A_{i, j}$ :

$\left(x_{i \pm 1 / 2}, y_{j-1 / 2}\right)$ and $\left(x_{i \pm 1 / 2}, y_{j+1 / 2}\right)$. This is done by computing $X\left(t^{n} ; t^{n+1},\left(x_{i}, y_{j}\right)\right)$ and $X\left(t^{n} ; t^{n+1},\left(x_{i \pm 1 / 2}, y_{j \pm 1 / 2}\right)\right)$ is deduced by interpolation. Hence, the computation of the feet of the characteristics is performed as in the standard semi-Lagrangian method. This determines a quadrilateral volume $a_{i, j} \approx X\left(t^{n} ; t^{n+1}, A_{i, j}\right)$ which will be supposed to be convex. This can be ensured providing that the time step $\Delta t$ is small enough.

The main step consists in the mesh intersection between the Lagrangian and Eulerian meshes. This step is not detailed here but we refer to [9].

The last step is the reconstruction step. Considering $a_{i, j}=\cup_{k, \ell=1} a_{i, j}^{k, \ell}$ where $a_{i, j}^{k, \ell}=a_{i, j} \cap A_{k, \ell}$, we rewrite (5) as

$$
\begin{aligned}
\bar{f}_{i, j}^{n+1} & =\frac{1}{\Delta x \Delta y} \int_{X\left(t^{n} ; t^{n+1}, A_{i, j}\right)} f^{n}(x, y) d x d y \\
& =\frac{1}{\Delta x \Delta y} \int_{a_{i, j}} f^{n}(x, y) d x d y \\
& =\frac{1}{\Delta x \Delta y} \sum_{k, \ell} \int_{a_{i, j}^{k, \ell}} f^{n}(x, y) d x d y
\end{aligned}
$$

From this last expression, we apply the Green theorem which reformulates an integral over a volume into an integral over segments. Then, it comes

$$
\begin{aligned}
& \frac{1}{\Delta x \Delta y} \sum_{k, \ell} \int_{a_{i, j}^{k, \ell}} f^{n}(x, y) d x d y= \\
& \int_{\partial a_{i, j}^{k, \ell}}[P(x, y) d x+Q(x, y) d y],
\end{aligned}
$$

where $\partial a_{i, j}^{k, \ell}$ denotes the boundary (which is composed of edges) of $a_{i, j}^{k, \ell}$ and $P, Q$ satisfy

$$
-\partial_{y} P(x, y)+\partial_{x} Q(x, y)=f^{n}(x, y)
$$


Finally, once the piecewise polynomial function has been determined, one can write

$$
f_{k, \ell}^{n}(x, y)=\sum_{p, q} c_{k, \ell}^{(p, q)} x^{p} y^{q}, \quad \forall(x, y) \in A_{i, j}
$$

so that

$$
\int_{a_{i, j}^{k, \ell}} f_{k, \ell}^{n}(x, y) d x d y=\sum_{p, q} c_{k, \ell}^{(p, q)} \int_{a_{i, j}^{k, \ell}} x^{p} y^{q} d x d y .
$$

We then have to compute the integral on the right hand side. To do that, we need some notations. For each subcell $a_{i, j}^{k, \ell}$, we introduce its corners $\left(x_{h}, y_{h}\right), h=0, \ldots, S-1$ and we suppose that $x_{0}=x_{S}, y_{0}=y_{S}$. Let us compute $\int_{a_{i, j}^{k, \ell}} x^{p} y^{q} d x d y$ for some given $(p, q)$ using the Green theorem. The case $p=q=1$ leads to

$$
\begin{aligned}
& \int_{a_{i, j}^{k, \ell}} x y d x d y= \sum_{h=1}^{S} \int_{\left(x_{h-1}, y_{h-1}\right)}^{\left(x_{h}, y_{h}\right)} \frac{x^{2} y}{2} d x d y \\
&= \frac{1}{2} \sum_{h=1}^{S} \int_{0}^{1}\left[x_{h-1}+t\left(x_{h}-x_{h-1}\right)\right]^{2} \\
& \times\left[y_{h-1}+t\left(y_{h}-y_{h-1}\right)\right] d t\left(y_{h}-y_{h-1}\right) \\
&= \frac{\left(y_{h}-y_{h-1}\right)}{2} \sum_{h=1}^{S}\left[y_{h-1}\left(3 x_{h-1}^{2}+2 x_{h-1} x_{h}+x_{h}^{2}\right)\right. \\
&\left.+y_{h}\left(x_{h-1}^{2}+2 x_{h-1} x_{h}+3 x_{h}^{2}\right)\right],
\end{aligned}
$$

where the changes of variables $x=x_{h-1}+t\left(x_{h}-x_{h-1}\right)$ and $y=y_{h-1}+t\left(y_{h}-y_{h-1}\right)$ have been done.

The algorithm of the conservative method to solve (2) then reads

- compute the 4 corners of $a_{i, j}$ by solving the characteristics ending at the 4 corners of $A_{i, j}$ backward in time,

- compute the intersection between the Lagrangian mesh and the cartesian axis,

- compute the corners of $a_{i, j}^{k, \ell}\left(\left(x_{h}, y_{h}\right), h=1, \ldots, S\right)$,

- compute the piecewise polynomial function using a Lagrange or Hermite reconstruction,

- compute $\int_{a_{i, j}^{k, \ell}} x^{p} y^{q} d x d y$ with the Green theorem,

- compute $\bar{f}_{i, j}^{n+1}$ with (7).

Standard numerical method We recall the main steps of the $2 D$ semi-Lagrangian method proposed in [20].

To go from a known function $f_{i, j}^{n}$ on the $2 D$ mesh $\left(x_{i}, y_{j}\right)$ to $f_{i, j}^{n+1}$, the semi-Lagrangian is based on the property that the solution is constant along the characteristics, and can be written as follows

- compute the feet of the characteristics $X\left(t^{n} ; t^{n+1},\left(x_{i}, y_{j}\right)\right)$, solution of $(3)$

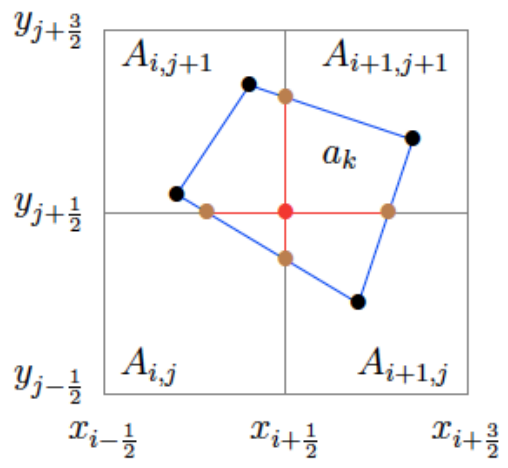

Fig. 1. Illustration of the intersection between the Lagrangian mesh $a_{k, \ell}$ and the Eulerian mesh $A_{i, j}$.

- compute

$$
\begin{aligned}
f_{i, j}^{n+1} & =f^{n}\left(X\left(t^{n} ; t^{n+1},\left(x_{i}, y_{j}\right)\right)\right) \\
& \simeq \mathcal{I}\left(f^{n}\right)\left(X\left(t^{n} ; t^{n+1},\left(x_{i}, y_{j}\right)\right)\right)
\end{aligned}
$$

where $\mathcal{I}$ is an interpolation operator (cubic splines for example) since $X\left(t^{n} ; t^{n+1},\left(x_{i}, y_{j}\right)\right)$ does not coincide with $\left(x_{i}, y_{j}\right)$ in general.

Extension to polar coordinates The two methods have been validated in classical test cases in cartesian configurations (using Vlasov-Poisson or guiding-center models). To deal with polar coordinates and non homogeneous Dirichlet boundary conditions, some adaptations are needed, similar to techniques proposed in [12] for the FSL method.

Since the new conservative method solves the conservative form of the transport equation, one has to consider the conservative solution $g(t, r, \theta)=r f(t, r, \theta)$ which is obtained by multiplying the solution by the Jacobian of the transformation. Then $g$ solves the following conservative form

$$
\partial_{t} g-\partial_{r}\left(\frac{\partial_{\theta} \Phi}{r} g\right)+\partial_{\theta}\left(\frac{\partial_{r} \Phi}{r} g\right)=0 .
$$

The conservative method is then applied to this equation. Since the BSL method solves the advective form, it does not require this adaptation.

Another point concerns boundary conditions of the unknown. To overcome this delicate issue, instead of working with $g$, we work with the unknown $\left(g-g_{\mathrm{eq}}\right)=r(f-$ $\left.f_{\text {eq }}\right)$ which satisfies homogeneous Dirichlet boundary conditions in our applications since the solution does not depart far from equilibrium. This is also applied to the standard BSL method.

\section{Diocotron instability test case}

This section is devoted to the presentation of the diocotron instability test case [15] which enables us to validate our new conservative method on a non trivial $2 D$ polar geometry and compare it to analytical results and to the BSL 
method. Let us mention the works [11] and [16], in which the guiding-center model is solved numerically in its polar version, using a slightly different initial condition.

We consider $\rho(t, r, \theta)$, a solution of $(1)$, with $\Phi(t, r, \theta)$ solving the Poisson equation:

$$
-\partial_{r}^{2} \Phi-\frac{1}{r} \partial_{r} \Phi-\frac{1}{r^{2}} \partial_{\theta}^{2} \Phi=\gamma \rho,
$$

where $\gamma \in\{-1,1\}$, according to the charge of the considered particles. The guiding-center model (1)-(9) satisfied by $\rho(t, r, \theta)$ is associated to an initial condition $\rho(t=$ $0, r, \theta)($ see $[7])$

$$
\rho(t=0, r, \theta)=\left\{\begin{array}{l}
0, r_{\min } \leq r<r_{-} \\
1+\epsilon \cos (\ell \theta), r_{-} \leq r<r_{+} \\
0, r_{+} \leq r \leq r_{\max }
\end{array}\right.
$$

where $\epsilon=10^{-6}$ (linear case) or $\epsilon=0.5$ (nonlinear case). We also need to define boundary conditions. In the $\theta \in$ $[0,2 \pi]$ direction, periodic boundary conditions are used so that a Fourier transform is applied to (9) whereas different conditions will be considered in the radial $r \in\left[r_{\min }, r_{\max }\right]$ direction. We recall the notation $\Omega=\left[r_{\min }, r_{\max }\right] \times[0,2 \pi]$.

\subsection{Radial boundary conditions for $\Phi$}

In this subsection, different radial boundary conditions are proposed to solve (9). In particular, their influence on the conservation of electric energy

$$
\mathcal{E}(t)=\int_{\Omega}\left[r\left|\partial_{r} \Phi\right|^{2}+\frac{1}{r}\left|\partial_{\theta} \Phi\right|^{2}\right] d r d \theta,
$$

and on total mass

$$
\mathcal{M}(t)=\int_{\Omega} r \rho d r d \theta
$$

is discussed.

First, homogeneous Dirichlet boundary conditions are imposed at $r_{\max }$ :

$$
\Phi\left(t, r_{\max }, \theta\right)=0, \forall t \geq 0, \theta \in[0,2 \pi] .
$$

Second, at $r_{\min }$, we consider two kinds of boundary conditions. We take either the homogeneous Dirichlet boundary condition

$$
\Phi\left(t, r_{\min }, \theta\right)=0, \forall t \geq 0, \theta \in[0,2 \pi],
$$

or the following condition, named Neumann mode 0:

- the homogeneous Neumann boundary condition at $r_{\min }$ for the Fourier mode 0 in $\theta\left(\partial_{r} \widehat{\Phi}_{0}\left(t, r_{\min }\right)=0\right)$

$$
\int_{0}^{2 \pi} \partial_{r} \Phi\left(t, r_{\min }, \theta\right) d \theta=0
$$

- the homogeneous Dirichlet boundary condition at $r_{\min }$ for the other Fourier modes $\left(\widehat{\Phi}_{k}\left(t, r_{\min }\right)=0, \forall k \neq 0\right)$

$$
\Phi\left(t, r_{\min }, \theta\right)=\frac{1}{2 \pi} \int_{0}^{2 \pi} \Phi\left(t, r_{\min }, \theta^{\prime}\right) d \theta^{\prime}
$$

For these two choices of radial boundary conditions, one can prove (see [15]) that the electric energy and the total mass are preserved in time

$$
\partial_{t} \mathcal{E}(t)=\partial_{t} \mathcal{M}(t)=0,
$$

which provides a good indicator to compare and validate the numerical methods. A last configuration has also been explored, in which homogeneous Neumann boundary conditions are imposed for all the modes $\partial_{r} \widehat{\Phi}_{k}\left(t, r_{\min }\right)=0, \forall k$. Let us remark that however in this case, the electric energy and the total mass are not preserved with time at the continuous level (see [15]).

For the solution $\rho$, Dirichlet type boundary conditions are considered:

$$
\begin{aligned}
& \rho\left(t, r \leq r_{\min }, \theta\right)=\rho\left(t, r_{\min }, \theta\right), \\
& \rho\left(t, r \geq r_{\max }, \theta\right)=\rho\left(t, r_{\max }, \theta\right) .
\end{aligned}
$$

\subsection{Instability rates}

In this subsection, the instability rates are computed from the linearized problem, considering the different radial boundary conditions.

Considering a radial equilibrium $\left(n_{0}(r), \Phi_{0}(r)\right)$ of (1)(9) linked by the Poisson equation

$$
-\partial_{r}\left(r \partial_{r} \Phi_{0}\right)=r n_{0},
$$

we then search for an approximate solution to(1)-(9) of the form

$$
\begin{aligned}
& \rho(t, r, \theta) \simeq n_{0}(r)+\epsilon \widehat{n}_{1, \ell}(r) \exp (\mathrm{i} \ell \theta) \exp (-\mathrm{i} \omega t / \gamma) \\
& \Phi(t, r, \theta) \simeq \gamma \Phi_{0}(r)+\epsilon \gamma \widehat{\Phi}_{1, \ell}(r) \exp (\mathrm{i} \ell \theta) \exp (-\mathrm{i} \omega t / \gamma) .
\end{aligned}
$$

Inserting this particular solution into (1)-(9) leads to the following system

$$
\left\{\begin{array}{l}
-\mathrm{i} \omega \widehat{n}_{1, \ell}-\mathrm{i} \ell \frac{\partial_{r} n_{0}}{r} \widehat{\Phi}_{1, \ell}+\mathrm{i} \ell \frac{\partial_{r} \Phi_{0}}{r} \widehat{n}_{1, \ell}=0 \\
-\partial_{r}^{2} \widehat{\Phi}_{1, \ell}-\frac{\partial_{r} \widehat{\Phi}_{1, \ell}}{r}+\frac{\ell^{2}}{r^{2}} \widehat{\Phi}_{1, \ell}=\widehat{n}_{1, \ell}
\end{array}\right.
$$

We express $\widehat{n}_{1, \ell}$ in terms of $\widehat{\Phi}_{1, \ell}$ to get an equation for $\widehat{\Phi}_{1, \ell}$ :

$$
\left(-\mathrm{i} \omega+\mathrm{i} \ell \frac{\partial_{r} \Phi_{0}}{r}\right)\left(-\partial_{r}^{2} \widehat{\Phi}_{1, \ell}-\frac{\partial_{r} \widehat{\Phi}_{1, \ell}}{r}+\frac{\ell^{2}}{r^{2}} \widehat{\Phi}_{1, \ell}\right)=\mathrm{i} \ell \frac{\partial_{r} n_{0}}{r} \widehat{\Phi}_{1, \ell}
$$

We can proceed in the numerical solution of the problem by making a discretization in the radial direction. With a uniform radial mesh $r_{i}=r_{\min }+i \Delta r, \Delta r=\left(r_{\max }-\right.$ $\left.r_{\text {min }}\right) / N_{r}$ and $N_{r} \in \mathbb{N}^{*}$, we introduce the following notations and approximations

$$
\begin{aligned}
\widehat{\Phi}_{1, \ell}\left(r_{i}\right) & =\phi_{i}, \\
\partial_{r} \widehat{\Phi}_{1, \ell}\left(r_{i}\right) & =\frac{\phi_{i+1}-\phi_{i-1}}{2 \Delta r} \\
\partial_{r}^{2} \widehat{\Phi}_{1, \ell}\left(r_{i}\right) & =\frac{\phi_{i+1}-2 \phi_{i}+\phi_{i-1}}{\Delta r^{2}} .
\end{aligned}
$$


Then, (14) is approximated by $A \phi=c B \phi$, with $c=\omega / \ell$ and where $\phi=\left(\phi_{0}, \phi_{1}, \ldots, \phi_{N}\right)^{T} \in \mathbb{R}^{N_{r}+1}$ and $A, B$ are two matrices of size $N_{r}+1$. The problem is then reduced to find the eigenvalues $c$ of the problem $B^{-1} A \phi=c \phi$. For each $\ell$, we look for the eigenvalue $c$ which has the greatest (strictly positive) imaginary part. We refer to [7] and [15] for more details.

\section{Linear case.}

Analytical growth rates $|\Im(\omega)|$ of the linear phase are obtained for all the discussed boundary conditions (the initial condition being (10) with $\epsilon=10^{-6}$ ) and comparison is made with the BSL method on a $128 \times 128$ grid, $\Delta t=0.05$, $r_{\min }=1, r_{\max }=10, \gamma=1$. In the following tables, comparisons between analytical and numerical growth rate for different modes $\ell$ and different $r_{ \pm}$are performed

- Table 1 refers to the first case (homogeneous Dirichlet boundary condition).

- Table 2 refers to the second case (Neumann mode 0).

- Table 3 refers to the third case (homogeneous Neumann boundary condition).

One can observe that the numerical instability rates are very close to the analytical ones (relative error of about $0.1 \%)$.

\section{Nonlinear case.}

Let us now consider the initial condition (10) with $\epsilon=0.5$ and study the influence of radial boundary conditions. To that purpose, we compare the new CSL method (the LAGH-9 method is used for the reconstruction) with the standard BSL method (cubic splines interpolation is used). Let us recall that the unknowns for CSL are centered at the vertices $r_{i}, \theta_{j}$, following (4).

We first focus on the Neumann mode 0 case: shown in Fig. 2 and 3 , we plot the time history of the electric energy $\mathcal{E}(t)$ and of the relative total mass $\left(\mathcal{M}(t)-\mathcal{M}_{e x}\right) / \mathcal{M}_{e x}$ (with $\left.\mathcal{M}_{\text {ex }}=\int_{r_{\min }}^{r_{\max }} \int_{0}^{2 \pi} \rho(t=0, r, \theta) r d r d \theta=28.27433389\right)$. A standard rectangle quadrature is used to approximate $\mathcal{E}$ and $\mathcal{M}$ given by (11) and (12). We compare the BSL method with different meshes and the new CSL method with $N_{r}=261, N_{\theta}=256$ and $\Delta t=0.1\left(N_{r}=261\right.$ is chosen to ensure that $r_{ \pm}$coincide with a mesh point). First, we can see that the new CSL method preserves the total mass better than the standard BSL method. Indeed, even with a refined mesh $N_{r}=1026, N_{\theta}=1024$ (purple curve in Fig. 3), the error in the BSL on this diagnostic is more important than the error in the CSL. The CSL method is not exactly conservative because of the boundary effect on $\rho$ : some loss or gain of mass can occur at $r_{\min }$ and $r_{\max }$. It is noted that the first order convergence of the trapezoidal quadrature rule in the $r$ direction: refining in this direction makes the relative total mass converge to zero at the initial time. Secondly, looking at the time evolution of the energy (see 2), the same conclusions are realised since CSL is very well behaved compared to BSL: the variation of total energy is about $3 \times 10^{-2}$ for CSL whereas for BSL, it is about $5 \times 10^{-2}$ using 16 for both.
Then, we focus on the Neumann case. We plot the same diagnostics in Fig. 4 and 5. In this Neumann configuration, the total mass and energy are not preserved at the continuous level, but it is interesting to observe how the numerical methods behave. Clearly, this is different since the CSL method does not preserve these two quantities as well as in the previous case (variations of about 0.4 for the total energy). However, it is difficult to define a good solution since the BSL method displays quite different behavior. A deeper analysis is needed and is not within the limits of this article.

\begin{tabular}{|c|c|c|c|c|}
\hline$\ell$ & $r_{-}$ & $r_{+}$ & $\Im(\omega)$ & analytical \\
\hline 2 & 4 & 5 & $0.2875, t \in[26,55]$ & 0.2887 \\
\hline 3 & 4 & 5 & $0.3667, t \in[26,72]$ & 0.3673 \\
\hline 4 & 4 & 5 & $0.3852, t \in[26,51]$ & 0.3840 \\
\hline 7 & 6 & 7 & $0.3424, t \in[26,61]$ & 0.3375 \\
\hline
\end{tabular}

Table 1. Growth rate obtained by the semi-Lagrangian method and by linearized analysis with homogeneous Dirichlet boundary conditions.

\begin{tabular}{|c|c|c|c|c|}
\hline$\ell$ & $r_{-}$ & $r_{+}$ & $\Im(\omega)$ & analytical \\
\hline 2 & 4 & 5 & $0.0719, t \in[93,138]$ & 0.0717 \\
\hline 3 & 4 & 5 & $0.2264, t \in[33,87]$ & 0.2267 \\
\hline 4 & 4 & 5 & $0.2991, t \in[34,69]$ & 0.2988 \\
\hline 7 & 6 & 7 & $0.3338, t \in[35,53]$ & 0.3307 \\
\hline
\end{tabular}

Table 2. Growth rate obtained by the semi-Lagrangian method and by linearized analysis with Neumann for mode 0 boundary conditions.

\begin{tabular}{|c|c|c|c|c|}
\hline$\ell$ & $r_{-}$ & $r_{+}$ & $\Im(\omega)$ & analytical \\
\hline 2 & 4 & 5 & $0.0672, t \in[89,151]$ & 0.0673 \\
\hline 3 & 4 & 5 & $0.2263, t \in[32,89]$ & 0.2266 \\
\hline 4 & 4 & 5 & $0.2991, t \in[34,69]$ & 0.2988 \\
\hline 7 & 6 & 7 & $0.3338, t \in[35,53]$ & 0.3307 \\
\hline
\end{tabular}

Table 3. Growth rate obtained by the semi-Lagrangian method and by linearized analysis with Neumann boundary conditions.

\section{Drift-kinetic Vlasov equation}

This last section is devoted to the validation of the new conservative method in the context of $4 D$ the drift-kinetic equation.

Indeed, we look for $f=f(t, r, \theta, z, v)$ satisfying the following $4 D$ slab drift-kinetic equation (see [10])

$$
\partial_{t} f-\frac{\partial_{\theta} \phi}{r} \partial_{r} f+\frac{\partial_{r} \phi}{r} \partial_{\theta} f+v \partial_{z} f-\partial_{z} \phi \partial_{v} f=0,
$$




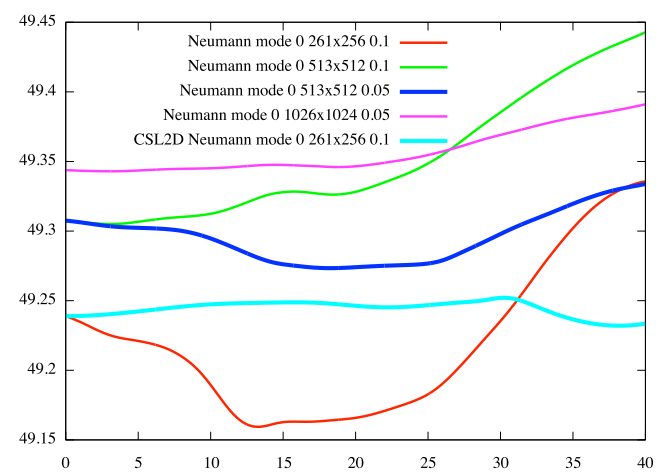

Fig. 2. Time evolution of $\mathcal{E}(t)$ for different grid sizes and $\Delta t$ : Neumann mode 0 case.

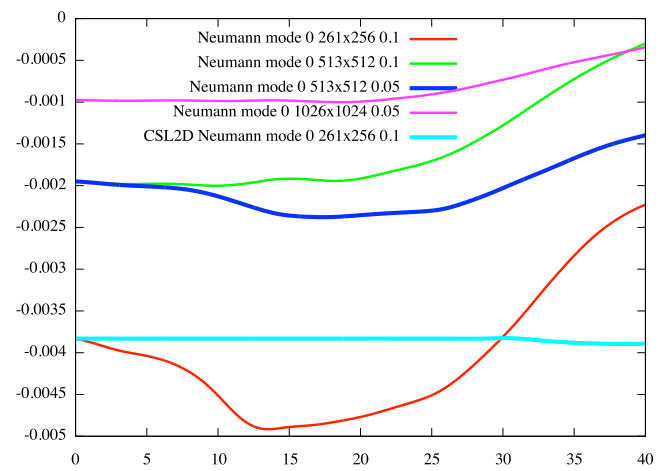

Fig. 3. Time evolution of the relative total mass $(\mathcal{M}(t)-$ $\left.\mathcal{M}_{e x}\right) / \mathcal{M}_{e x}$ for different grid sizes and $\Delta t$ : Neumann mode 0 case.

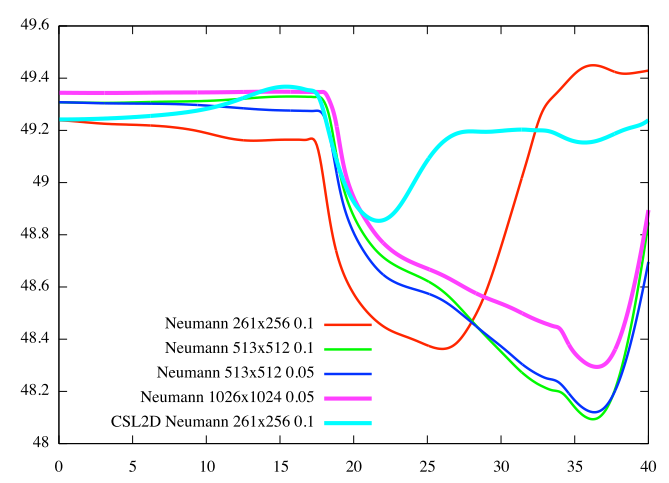

Fig. 4. Time evolution of $\mathcal{E}(t)$ for different grid sizes and $\Delta t$ : Neumann case.

for $(r, \theta, z, v) \in \Omega \times[0, L] \times \mathbb{R}$. The self-consistent potential $\phi=\phi(r, \theta, z)$ solves the quasi neutrality equation

$$
\begin{gathered}
-\left[\partial_{r}^{2} \phi+\left(\frac{1}{r}+\frac{\partial_{r} n_{0}(r)}{n_{0}(r)}\right) \partial_{r} \phi+\frac{1}{r^{2}} \partial_{\theta}^{2} \phi\right]+\frac{1}{T_{e}(r)}(\phi-\langle\phi\rangle) \\
=\frac{1}{n_{0}(r)} \int_{\mathbb{R}} f d v-1,
\end{gathered}
$$

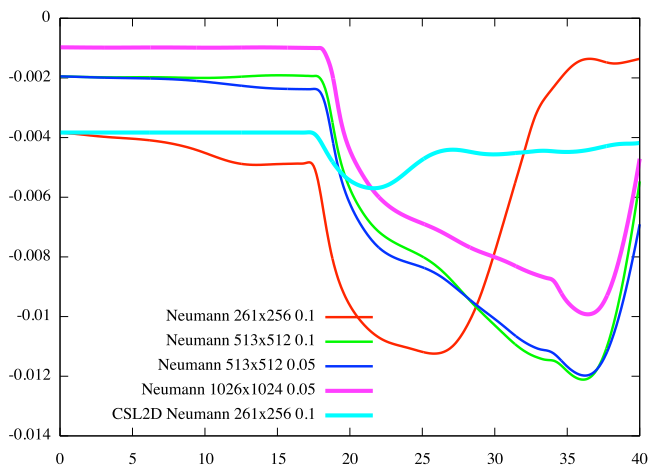

Fig. 5. Time evolution of the relative total mass $(\mathcal{M}(t)-$ $\left.\mathcal{M}_{e x}\right) / \mathcal{M}_{e x}$ for different grid sizes and $\Delta t$ : Neumann case.

with $\langle\phi\rangle=\frac{1}{L} \int_{0}^{L} \phi(r, \theta, z) d z$. Periodic boundary conditions are considered in the $\theta$ and $z$ directions whereas in the radial direction, Neumann mode 0 is imposed (see [13]). For the solution $f$, periodic boundary conditions are imposed in the $\theta, z, v$ directions, whereas in the radial $r$ directions, Dirichlet boundary conditions are considered.

The initial function is given by

$$
\begin{aligned}
& f(t=0, r, \theta, z, v)= \\
& \quad f_{\text {eq }}(r, v)\left[1+\epsilon \exp \left(-\frac{\left(r-r_{p}\right)^{2}}{\delta r}\right) \cos \left(\frac{2 \pi n}{L} z+m \theta\right)\right],
\end{aligned}
$$

where the equilibrium function is

$$
f_{\mathrm{eq}}(r, v)=\frac{n_{0}(r) \exp \left(-\frac{v^{2}}{2 T_{i}(r)}\right)}{\left(2 \pi T_{i}(r)\right)^{1 / 2}} .
$$

The radial profiles $\left\{T_{i}, T_{e}, n_{0}\right\}$ have the analytical expressions

$\mathcal{P}(r)=C_{\mathcal{P}} \exp \left(-\kappa_{\mathcal{P}} \delta r_{\mathcal{P}} \tanh \left(\frac{r-r_{p}}{\delta r_{\mathcal{P}}}\right)\right), \mathcal{P} \in\left\{T_{i}, T_{e}, n_{0}\right\}$,

where the constants are

$C_{T_{i}}=C_{T_{e}}=1, C_{n_{0}}=\frac{r_{\max }-r_{\min }}{\int_{r_{\min }}^{r_{\max }} \exp \left(-\kappa_{n_{0}} \delta r_{n_{0}} \tanh \left(\frac{r-r_{p}}{\delta r_{n_{0}}}\right)\right) d r}$.

Finally, we consider the parameters of [4] (MEDIUM case)

$$
\begin{aligned}
& r_{\min }=0.1, r_{\max }=14.5, \\
& \kappa_{n_{0}}=0.055, \kappa_{T_{i}}=\kappa_{T_{e}}=0.27586, \\
& \delta r_{T_{i}}=\delta r_{T_{e}}=\frac{\delta r_{n_{0}}}{2}=1.45, \epsilon=10^{-6}, n=1, m=5, \\
& L=1506.759067, r_{p}=\frac{r_{\min }+r_{\max }}{2}, \delta r=\frac{4 \delta r_{n_{0}}}{\delta r_{T_{i}}} .
\end{aligned}
$$

\subsection{Global algorithm}

To deal with the $4 D$ drift-kinetic model, a directional splitting is performed as in [10]. As said before, the standard semi-Lagrangian method and the new conservative 
semi-Lagrangian method have common steps, in particular because in the $1 D z$ and $v$ configuration, we deal with the constant advection coefficient for which the BSL and CSL methods are equivalent (see [6]). The only difference between the two methods lies in the $2 D$ advection in $(r, \theta)$. We then call BSL2D the method proposed in [10] and CSL2D the new conservative method.

The main steps of the algorithms BSL2D and CSL2D are

- solve the $1 D$ advection $\partial_{t} f+v \partial_{z} f=0$ on $\Delta t / 2$ with the standard semi-Lagrangian method

(periodic cubic splines interpolation),

- solve $1 D$ advection $\partial_{t} f-\partial_{z} \Phi \partial_{v} f=0$ on $\Delta t / 2$ with the standard semi-Lagrangian method

(periodic cubic splines interpolation),

- solve the quasi neutrality equation (16) to compute the potential $\Phi$, with FFT in $\theta, z$ and second order finite difference in $r$,

- compute the derivatives $\left(\partial_{r} \Phi, \partial_{\theta} \Phi, \partial_{z} \Phi\right)$ of $\Phi$ with cubic splines in $r, \theta$ and second order finite differences in $z$,

- $2 D$ advection in $(r, \theta)(1)$, with BSL or CSL,

- solve $1 D$ advection $\partial_{t} f-\partial_{z} \Phi \partial_{v} f=0$ on $\Delta t / 2$ with the standard semi-Lagrangian method

(periodic cubic splines interpolation),

- solve the $1 D$ advection $\partial_{t} f+v \partial_{z} f=0$ on $\Delta t / 2$ with the standard semi-Lagrangian method (periodic cubic splines interpolation).

\subsection{Numerical results}

In this section, we compare the standard BSL method to the new conservative method. To do that, we consider the $4 D$ drift-kinetic model in polar geometry (15)-(16). The practical implementation is done in a modular semiLagrangian library [19].

We are interested in the time history of

$$
\sqrt{\int \Phi^{2}\left(r_{p}, \theta, z\right) d \theta d z}
$$

where $r_{p}=\left(r_{\max }+r_{\min }\right) / 2$, of the total mass

$$
\mathcal{M}(t)=\int_{r_{\min }}^{r_{\max }} \int_{0}^{2 \pi} \int_{0}^{L} \int_{\mathbb{R}} f(t, r, \theta, z, v) r d v d z d \theta d r
$$

and of the total energy

$$
\begin{aligned}
& \mathcal{E}(t)=\int_{r_{\min }}^{r_{\max }} \int_{0}^{2 \pi} \int_{0}^{L} \int_{\mathbb{R}} \frac{v^{2}}{2} f(t, r, \theta, z, v) r d v d z d \theta d r \\
& +\int_{r_{\min }}^{r_{\max }} \int_{0}^{2 \pi} \int_{0}^{L} \int_{\mathbb{R}} f(t, r, \theta, z, v) \Phi(t, r, \theta, z) r d v d z d \theta d r .
\end{aligned}
$$

The first quantity (17) is known to exponentially increase with time. The growth rate can be obtained by a generalization of the approach performed in [4]. Hence, growth rates obtained in [4] can be compared to numerical results. The total mass $\mathcal{M}(t)$ is preserved by the model

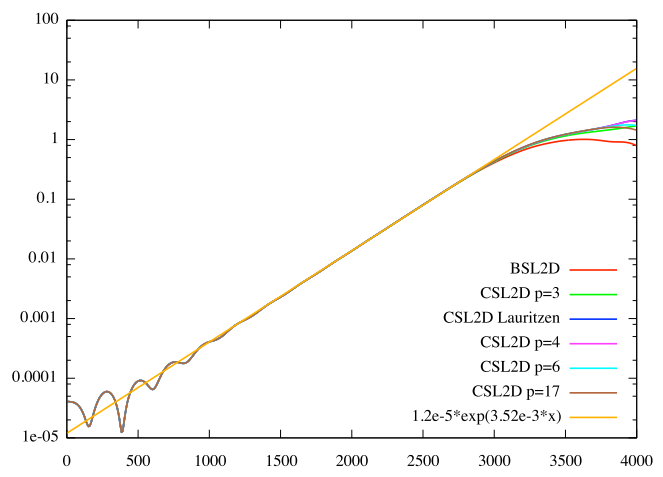

Fig. 6. Time evolution of $\sqrt{\int \Phi^{2}\left(r_{p}, \theta, z\right) d \theta d z}$ on $32 \times 32 \times$ $32 \times 64$ grid, with $\Delta t=8$ and $v_{\max }=7.32$.

and it is an important point for the methods to preserve this quantity as much as possible.

In Fig. 6, we plot the time history of (17) obtained with BSL2D and CSL2D. Different variants of the CSL2D method are shown, corresponding to different reconstructions presented in section $2: p=3$ which corresponds to third order Lagrange reconstruction, $p=4$ (resp. 6) which corresponds to PPM (resp. PPM2), $p=17$ which corresponds to LAGH17, and Lauritzen which corresponds to the reconstruction proposed in [14]. One can observe that the linear part is well reproduced by all the method since the instability rate of all the methods is very close to the analytical one proposed by [4].

In Fig. 7, the time history of the total mass $\mathcal{M}(t)$ (which is theoretically preserved with time) is plotted for the different methods (BSL2D and CSL2D for different reconstructions). The final time is 8000 chosen so that the linear theory is not available any more. We can first observe that up to time 3000 , the total mass is well preserved by all methods. This time interval corresponds to the linear phase in which few phenomena occur. After that time, the methods behave differently. In particular, BSL2D looses the conservation of total mass (even with a relative error of about $0.1 \%$ ). On the other side, CSL2D presents better behavior (relative error of about $0.01 \%$ ). Among the different reconstructions, CSL2D $p=6$ (which corresponds to PPM2) behaves in the best way.

Fig. 8 shows the time history of $\mathcal{E}(t)$ (which is theoretically preserved with time) obtained with the different methods. This invariant is quite difficult to preserve numerically and we can observe that, as for the total mass, the CSL methods display very good behavior compared to the BSL method since the total energy is better conserved (about $0.1 \%$ for CSL and about $1 \%$ for BSL). One interesting point is the very similar behavior of the total mass and total energy. It seems that the loss of the mass conservation has a strong influence on the total energy conservation. Obviously we have to go further into the comparisons but these first results are quite promising. 


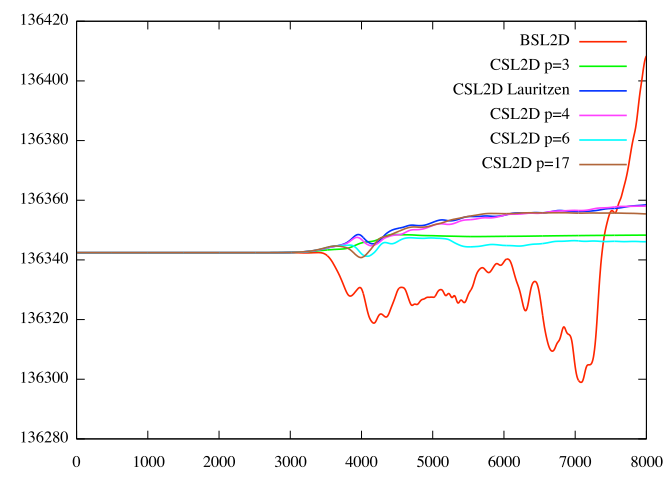

Fig. 7. Time evolution of the total mass on $32 \times 32 \times 32 \times 64$ grid, with $\Delta t=8$ and $v_{\max }=7.32$.

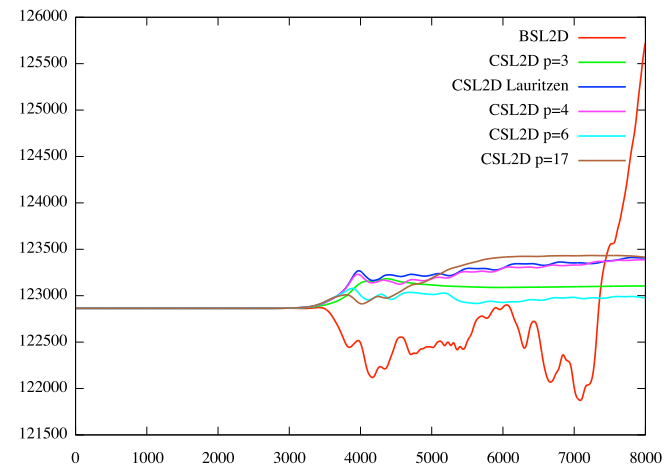

Fig. 8. Time evolution of the total energy on $32 \times 32 \times 32 \times 64$ grid, with $\Delta t=8$ and $v_{\max }=7.32$.

\section{Conclusion}

In this work, we proposed the validation of a new conservative semi-Lagrangian method CSL, on two non trivial test cases in polar geometry. The first test concerns a guiding-center model for which boundary conditions in the radial directions are discussed. It appears that some good choices lead to conservation of electric energy and total mass. This is confirmed by the numerical results in which we observe a very good behavior of the new method compared to BSL. The second test concerns a $4 D$ drift-kinetic model which can be viewed as a first step to describe tokamak plasmas. For these two models, a linear analysis can be performed and instability rates can be obtained to validate the numerical methods in the linear phase.

The new CSL method seems to be a good alternative to the standard pointwise semi-Lagrangian method which is not conservative in these configurations. More comparisons are performed in [9] and [5] in these two intermediate configurations (guiding-center and $4 D$ drift-kinetic) to confirm the good behavior of the conservative semiLagrangian method.

\section{References}

1. C.K. Birdsall, A.B. Langdon, Plasma Physics via Computer Simulation, Inst. of Phys. Publishing, Bristol/Philadelphia, 1991.

2. C.Z. Cheng, G. Knorr, The integration of the Vlasov equation in configuration space, J. Comput. Phys. 22, (1976), pp. 330-3351.

3. P. Colella, P. Woodward, The Piecewise Parabolic Method (PPM) for gas-dynamical simulations, J. Comput. Phys. 54, (1984), pp. 174-201.

4. D. Coulette, N. Besse, Numerical comparisons of gyrokinetic multi-water-bag models, J. Comput. Phys. 248, (2013), pp. 132.

5. N. Crouseilles, P. Glanc, M. Mehrenberger, 2D advective and conservative semi-Lagrangian schemes upwind Hermite type reconstruction, in preparation.

6. N. Crouseilles, M. Mehrenberger, E. Sonnendrücker, Conservative semi-Lagrangian method for the Vlasov equation, J. Comput. Phys. 229, (2010), pp. 1927-1953.

7. R.C. Davidson, Physics of non neutral plasmas, AddisonWesley, Redwood City, CA,1990.

8. R. Gagné, M. Shoucri, A splitting scheme for the numerical solution of a one-dimensional Vlasov equation, J. Comput. Phys. 24, (1977), pp. 445-449.

9. P. Glanc, Approximation numérique de l'équation de Vlasov par des méthodes de type remapping conservatif, $\mathrm{PhD}$ of the University of Strasbourg, 2014.

10. V. Grandgirard, M. Brunetti, P. Bertrand, N. Besse, X. Garbet, P. Ghendrih, G. Manfredi, Y. Sarazin, O. Sauter, E. Sonnendrücker, J. Vaclavik, L. Villard, A drift-kinetic SemiLagrangian $4 D$ code for ion turbulence simulation, J. Comput. Phys. 217, (2006), pp. 395-423.

11. B. Krane, I. Christopher, M. Shoucri, G. Knorr, Suppression of coherent structures in sheared plasma flows, Phys. Rev. Lett. 80, (1998), pp. 4422-4425.

12. G. Latu, V. Grandgirard, J. Abiteboul, M. Bergot, N. Crouseilles, X. Garbet, P. Ghendrih, M. Mehrenberger, Y. Sarazin, H. Sellama, E. Sonnendrücker, D. Zarzoso, Accuracy of unperturbed motion of particles in a gyrokinetic semiLagrangian code, INRIA Research Report 8054.

13. G. Latu, V. Grandgirard, J. Abiteboul, N. Crouseilles, G. Dif-Pradalier, X. Garbet, P. Ghendrih, M. Mehrenberger, Y. Sarazin, E. Sonnendrücker, D. Zarzoso, Improving conservation properties in a $5 \mathrm{D}$ gyrokinetic semi-Lagrangian code, submitted.

14. P. Lauritzen, D. Ramachandran, P. Ullrich, A conservative semi-Lagrangian multi-tracer transport scheme (CSLAM) on the cubed-sphere grid, J. Comput. Phys. 229, (2010), pp. 1401-1424.

15. E. Madaule, S. Hirstoaga, M. Mehrenberger, J. Pétri, Semi-Lagrangian simulations of the diocotron instability, hal00841504 .

16. R. Marchand, M. Shoucri, Numerical simulation of the excitation and evolution of high-azimuthal-mode-number coherent vortices in hollow magnetized electron columns, J. Plasma Phys. 65, (2001), pp. 151-160.

17. J. Pétri, The diocotron instability in a pulsar "cylindrical" electrosphere, Astronomy \& Astrophysics, 2008.

18. J. Pétri, Non-linear evolution of the diocotron instability in a pulsar electrosphere: 2D PIC simulations, Astronomy \& Astrophysics, 2009.

19. SELALIB, http://selalib.gforge.inria.fr/ 
20. E. Sonnendrücker, J. Roche, P. Bertrand, A. Ghizzo, The Semi-Lagrangian Method for the Numerical Resolution of the Vlasov Equation, J. Comput. Phys. 149, 201-220 (1999).

21. C. Yang, F. Filbet, Conservative and non-conservative methods based on Hermite weighted essentially-nonoscillatory reconstruction for Vlasov equations, submitted, http://arxiv.org/pdf/1312.4481v1.pdf 